\title{
Full-wave inversion in frequency-domain
}

\author{
Shin'ichi Uesaka \\ Kansai Research Institute, Inc., Japan.
}

\begin{abstract}
We made a numerical experiment on full-wave inversion based on acoustic wave-equation in frequency-domain. The core of this method is that we can select frequencies to calculate the Frechét derivative of wave-field in terms of velocity at each iteration. Numerical experiments show that we don't have to use all frequencies in the observed data at one iteration. In the early stage of iteration we calculate the Frechét derivative by using low frequency, and later by using high frequency. Consequently computing cost is lower than that by using all frequencies and we can get a high-resolution image.
\end{abstract}

\section{INTRODUCTION}

Full wave inversion methods can be classified to two main methods; The time-domain method (TARANTOLA, 1984) and the frequency-domain method (PrATT, 1990). We have investigated full-wave inversion methods based on the acoustic wave-equation in time domain (WATANABE et al, 1997). In the time-domain method, the data used for inversion are waveforms. The forward and the backward modeling are performed by solving the acoustic wave-equation in time-domain. In the frequency-domain method, the data are spectrum. The modeling is performed solving the equation in frequency-domain.

The purpose of this study is to examine the characteristics, the advantages and the disadvantages of the acoustic full-wave inversion in frequency-domain. In the first section, we briefly present the theory of the acoustic full-wave inversion in frequency-domain. In the second section we investigate the effect of a frequency for inversion on the reconstructed image by computational studies.

\section{THEORY}

The acoustic wave equation in frequency-domain is described as follows;

$$
\frac{\omega^{2}}{c(\boldsymbol{x})^{2}} P\left(\boldsymbol{x}, \omega ; \boldsymbol{x}_{s}\right)+\nabla^{2} P\left(\boldsymbol{x}, \omega ; \boldsymbol{x}_{s}\right)=-S\left(\omega ; \boldsymbol{x}_{s}\right)
$$


where, $\omega$ is the angular frequency, $c(x)$ is the wave velocity, $P$ and $S$ are the complex spectrum of the pressure field and the source function at location $\boldsymbol{x}_{\boldsymbol{s}}$ respectively. The full-wave inversion in frequency-domain attempts to determine the velocity model, $c(\boldsymbol{x})$, that minimizes the following error function:

$$
E=\frac{1}{2} \sum_{s} \sum_{r} \int \delta P\left(\boldsymbol{x}_{r}, \omega ; \boldsymbol{x}_{s}\right)^{2} d \omega
$$

where, $\delta P\left(\boldsymbol{x}_{\boldsymbol{r}}, \omega ; \boldsymbol{x}_{\boldsymbol{s}}\right)$ is the data residuals defined by

$$
\delta P\left(\boldsymbol{x}_{r}, \omega ; \boldsymbol{x}_{s}\right)=P\left(\boldsymbol{x}_{r}, \omega ; \boldsymbol{x}_{s}\right)_{o b s}-P\left(\boldsymbol{x}_{r}, \omega ; \boldsymbol{x}_{s}\right)_{f o r}
$$

where, $P\left(\boldsymbol{x}_{r}, \omega ; \boldsymbol{x}_{s}\right)_{o b s}$ is the complex spectrum of the observed data and $P\left(\boldsymbol{x}_{r}, \omega ; \boldsymbol{x}_{s}\right)_{f o r}$ is that of the calculated data for the current velocity model.

We used the steepest descent method to find the velocity model that minimizes the error function, $E$. To update the velocity model, the steepest descent method gives;

$$
c(\boldsymbol{x})_{k+1}=c(\boldsymbol{x})_{k}+\alpha_{k} \Gamma(\boldsymbol{x})
$$

where, $\Gamma(\boldsymbol{x})_{k}$ is the steepest descent direction of the error function, $E$, and $\alpha_{k}$ is the step length for the $k$ th iteration. $\Gamma(\boldsymbol{x})$ is calculated by

$$
\Gamma(\boldsymbol{x})=\frac{1}{c(\boldsymbol{x})^{3}} \int \Gamma(\boldsymbol{x}, \omega) d \omega
$$

where

$$
\Gamma(\boldsymbol{x}, \omega)=\sum_{s} P\left(\boldsymbol{x}, \omega ; \boldsymbol{x}_{s}\right)_{f o r} P\left(\boldsymbol{x}, \omega ; \boldsymbol{x}_{s}\right)_{b a c k}
$$

$P\left(\boldsymbol{x}, \omega ; \boldsymbol{x}_{s}\right)_{f o r}$ is the pressure field calculated for the current velocity model. $P\left(\boldsymbol{x}, \omega ; \boldsymbol{x}_{s}\right)_{\text {back }}$ is calculated by

$$
P\left(\boldsymbol{x}, \omega ; \boldsymbol{x}_{s}\right)_{b a c k}=\sum_{\boldsymbol{r}} G\left(\boldsymbol{x}, \boldsymbol{x}_{r}\right) \delta P^{*}\left(\boldsymbol{x}_{\boldsymbol{r}}, \omega ; \boldsymbol{x}_{s}\right)
$$

where, $G\left(\boldsymbol{x}, \boldsymbol{x}_{\boldsymbol{r}}\right)$ is the Green's function of the acoustic wave equation in frequency-domain and the asterisk represents the complex conjugate. By equation (7), the data residuals propagate from the receiver location $\boldsymbol{x}_{\boldsymbol{r}}$ into the model space. This is so called "backpropagation" in frequency-domain.

\section{NUMERICAL EXPERIMENT}

Figure 1 shows the "true" velocity model from which we calculate the observed data by using a finite-difference method in frequency domain. The calculation area is $90 \mathrm{~m}$ wide and $90 \mathrm{~m}$ deep. The object area is $80 \mathrm{~m}$ wide and $80 \mathrm{~m}$ deep. 41 sources are evenly spaced in the left side of the object area, and 41 receivers are evenly spaced in the right side of the object area. The source wave is the Ricker wavelet of which the peak frequency is $100 \mathrm{~Hz}$. Figure 2 shows the spectrum of the ovserved data of the common shot gather in terms of 21 st source. The observed data were calculated for the "true" velocity model. 
In this case, $\Delta x=\Delta z=1.0 \mathrm{~m}, \Delta t=0.78125 \mathrm{~ms}$ and 128 time steps are calculated. In other word, the frequency step, $\Delta f$, is $10 \mathrm{~Hz}$. (a), the left panel is the real part of the spectrum and the right panel is the imaginary part. (b) is the waveform in time domain.

We considered two cases. In case1 $\Gamma(x)$ is calculated by using 24 frequencies from 10 $\mathrm{Hz}$ to $240 \mathrm{~Hz}$ at the intervals of $10 \mathrm{~Hz}$ for each iteration. In case2, $\Gamma(x)$ is calculated by one frequency only. We started from a low frequency, for first iteration, $10 \mathrm{~Hz}$. The frequency is gradually increased at the intervals of $10 \mathrm{~Hz}$ to $240 \mathrm{~Hz}$ for 24 th iteration. The initial model for both cases is homogeneous model of $3000 \mathrm{~m} / \mathrm{s}$. Figure 3 shows the data residual calculated with respect to the initial model to the observed data. Figure 4 shows the reconstructed velocity model of each case after 24 iterations. Figure 5 shows the error function at each 6 iterations. Figure 6 shows the data residual calculated with respect to the final result of case 2 to the observed data. The result of case 2 is more accurate than that of case1.

\section{CONCLUSIONS}

We study on the acoustic full-wave inversion in frequency-domain. One of advantages of this method is that we can select the frequency for the inversion at each iteration. We showed by numerical experiments that we could get high-resolution velocity image by using one frequency component for one iteration. In early stage of iteration, we can calculate the Frechét derivative by using low frequencies and later by using high frequencies. Consequently the computing cost is lower than that by using all frequencies and we can get a high-resolution image.

\section{ACKNOWLEGMENTS}

The author is grateful to Dr. Yuzuru Ashida, Dr. Toshifumi Matsuoka and Dr Toshiki Watanabe for kind and fruitful discussion which improve this reserch.

\section{REFERENCE}

TARANTOLA, A. (1984): Inversion of seismic reflection data in the acoustic approximation, Geophisics, 39, 1259-1266

Pratt, R. G. and Worthington, M. H. (1990): Inverse theory applied to multisource cross-hole tomography, Part I: Acoustic wave-equation method, Geophys. Prosp., 38, 287-310.

Watanabe, T., Uesaka, S., Sassa, K. (1997): A computational study on full-wave inversion based on the acoustic wave-equation (1)-Evaluation of inversion methods-, BUTSURI-TANSA, 50, 197-208. 


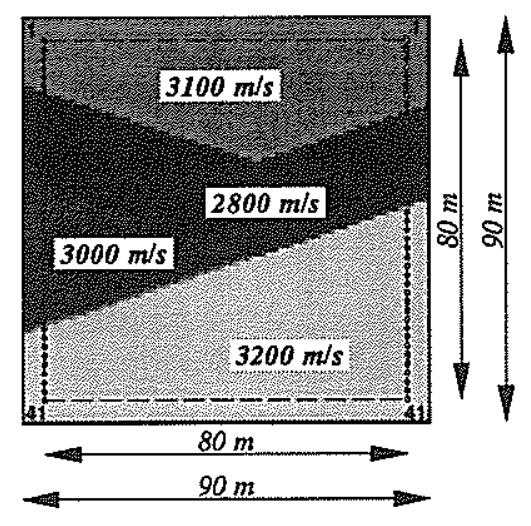

Figure 1: Calculation area, object area and velocity structure of the "true" model used for the numerical experiments.
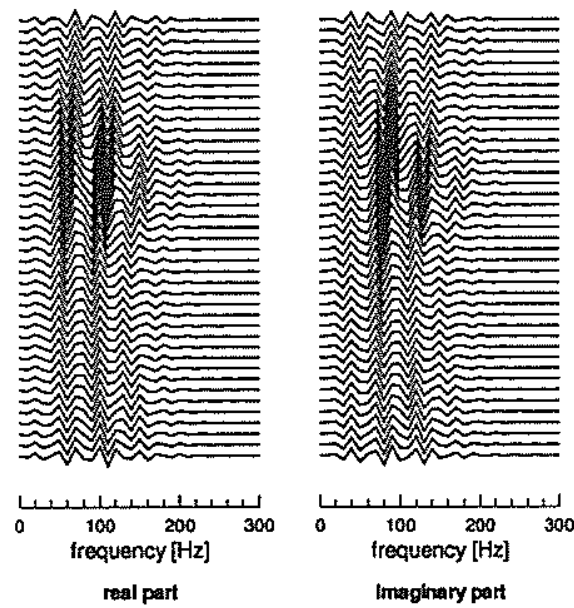

(a)

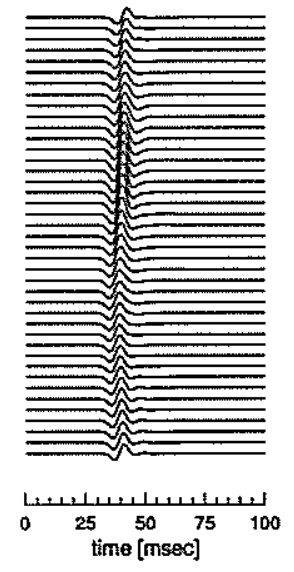

(b)

Figure 2: Common shot gather of the observed data. (a) The wave spectrum in frequency-domain. The left panel is the real part, and the right panel is the imaginary part. (b) The waveform in time-domain. The source number is 21. 

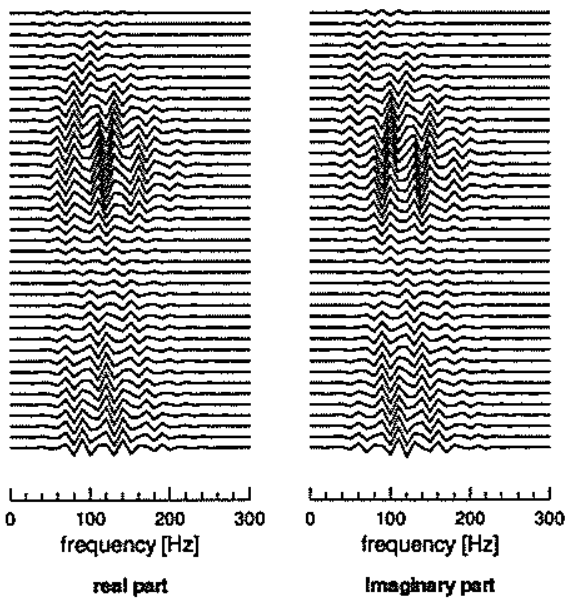

(a)

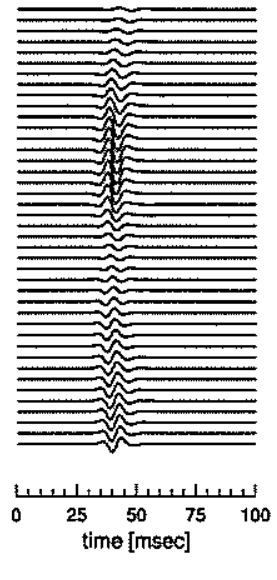

(b)

Figure 3: Residuals of the calculated data with respect to the initial model to the observed data. (a) The wave spectrum in frequency-domain. The left panel is the real part, and the right panel is the imaginary part. (b) Waveform in time-domain. The source number is 21 .
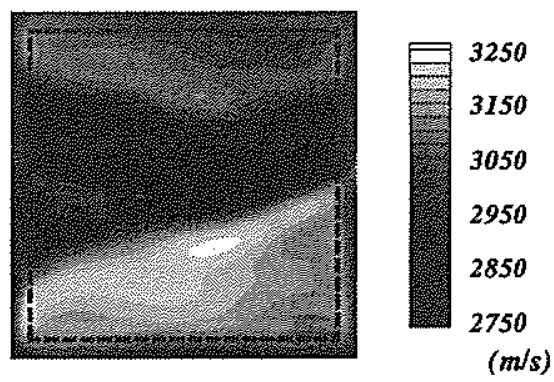

(a) case-1

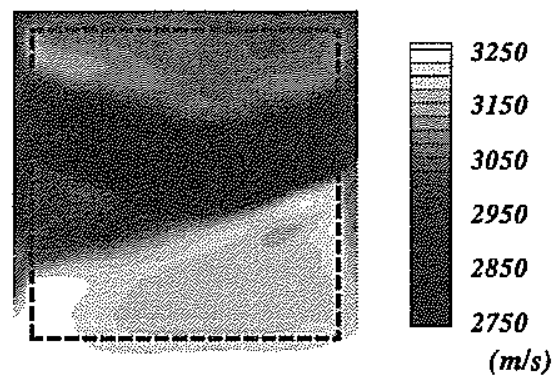

(c) case-2

Figure 4: Reconstructed velocity image of (a) case1 and (b) case 2 after 24 iterations. 


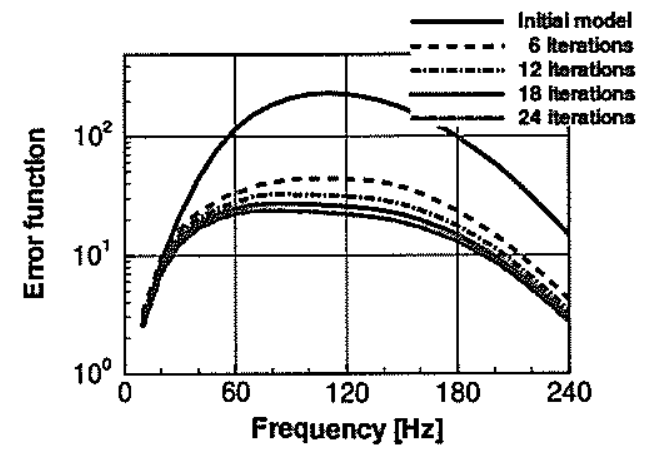

(a) case1

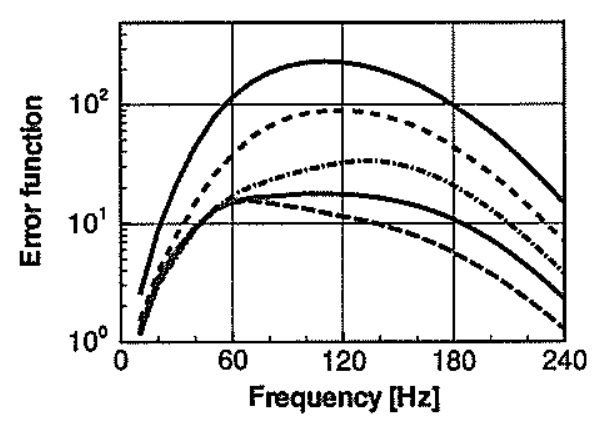

(c) case2

Figure 5: Error function of (a) case1, (b) case2 at each 6 iterations.

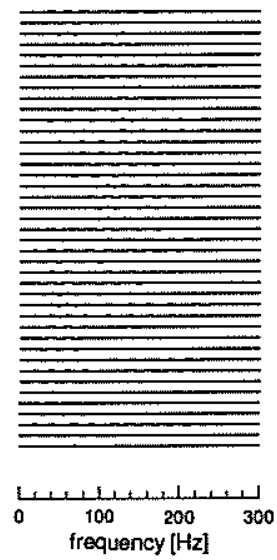

real part

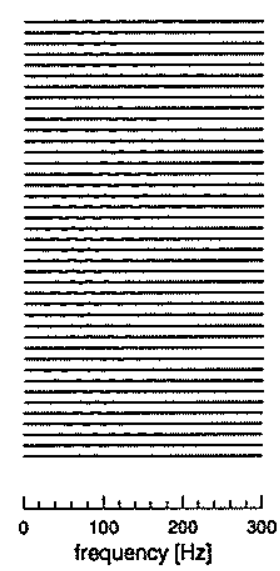

Imagirary part

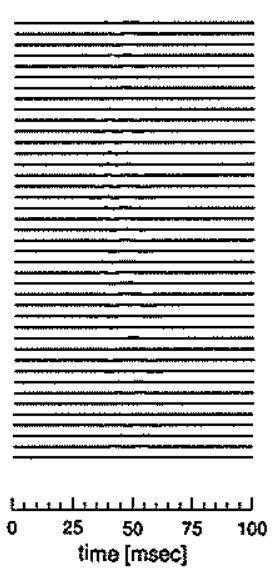

(b)

Figure 6: Residuals of the calculated data with respect to the reconstructed model of case 2 to the observed data. (a) Wave spectrum in frequency-domain. The left panel is the real part, and the right panel is the imaginary part. (b) Waveform in time-domain. The source number is 21 . 